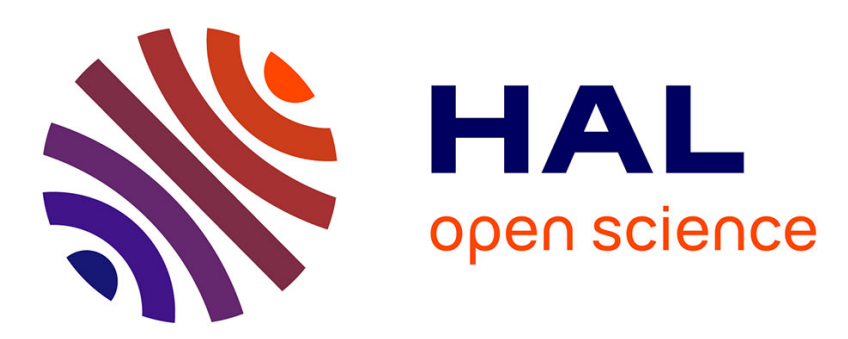

\title{
Dépolarisation de la lumière diffusée par des molécules anisotropes: dépendance avec la densité
}

\author{
J. Berrue, A. Chave, B. Dumon, M. Thibeau
}

\section{To cite this version:}

J. Berrue, A. Chave, B. Dumon, M. Thibeau. Dépolarisation de la lumière diffusée par des molécules anisotropes: dépendance avec la densité. Journal de Physique Lettres, 1976, 37 (7-8), pp.165-168. 10.1051/jphyslet:01976003707-8016500 . jpa-00231265

\section{HAL Id: jpa-00231265 https://hal.science/jpa-00231265}

Submitted on 1 Jan 1976

HAL is a multi-disciplinary open access archive for the deposit and dissemination of scientific research documents, whether they are published or not. The documents may come from teaching and research institutions in France or abroad, or from public or private research centers.
L'archive ouverte pluridisciplinaire HAL, est destinée au dépôt et à la diffusion de documents scientifiques de niveau recherche, publiés ou non, émanant des établissements d'enseignement et de recherche français ou étrangers, des laboratoires publics ou privés. 


\title{
DÉPOLARISATION DE LA LUMIÈRE DIFFUSÉE PAR DES MOLÉCULES ANISOTROPES : DÉPENDANCE AVEC LA DENSITÉ
}

\author{
J. BERRUE, A. CHAVE, B. DUMON et M. THIBEAU
}

L.E.I.M.D.L., Faculté des Sciences, 49045 Angers Cedex, France

(Reçu le 27 février 1976, accepté le 3 mai 1976)

\begin{abstract}
Résumé. - Trappeniers et coll. [1] calculent le taux de dépolarisation de la lumière diffusée dans l'approximation D.I.D. et font l'hypothèse de l'orientation aléatoire des axes intermoléculaires des molécules en cours de collisions. Cette hypothèse n'est satisfaisante qu'à très basse densité $[2,3]$. Nous tenons compte des corrélations d'orientations des axes intermoléculaires et donnons une expression du taux de dépolarisation pour des molécules faiblement anisotropes à densités modérées. L'accord avec les mesures $\left(\mathrm{N}_{2}, \mathrm{C}_{2} \mathrm{H}_{4}\right)$ [4] est satisfaisant.
\end{abstract}

\begin{abstract}
Trappeniers et al. [1], using a D.I.D. approximation, have given an expression of the depolarization of scattered light. They assume that the orientations of the intermolecular axes of pairs of colliding molecules are independent. Except at very low density, this hypothesis is not satisfactory $[2,3]$. In this paper we take into account the correlations of the orientation of these intermolecular axes and we give a theoretical expression valid for weakly anisotropic molecules at moderate densities. The results obtained are in good agreement with experimental data $\left(\mathrm{N}_{2}, \mathrm{C}_{2} \mathrm{H}_{4}\right)$ [4].
\end{abstract}

Un fluide est composé de molécules linéaires. Leurs positions sont repérées par un trièdre orthonormé $O x y z$. Un faisceau polarisé selon $O z$ se propage selon $x$ et on observe les intensités diffusées selon $O y\left(I_{x z}\right.$ et $\left.I_{z z}\right)$ polarisées selon $x$ et $z$. La matrice $(\alpha)$ de polarisabilité d'une molécule peut s'écrire :

$$
(\alpha)=\bar{\alpha}(U)+(\beta)
$$

$(U)$ est la matrice identité, $\bar{\alpha}$ la polarisabilité moyenne, $(\beta)$ une matrice à trace nulle dont les éléments sont liés à l'orientation de la molécule et à son anisotropie $\delta$

$$
\cdot \bar{\alpha}=\frac{\alpha_{\|}+2 \alpha_{\perp}}{3}, \quad \delta=\alpha_{\|}-\alpha_{\perp} .
$$

Nous supposerons $(\alpha)$ non modifié par les collisions (approximation D.I.D.). Selon un calcul traditionnel [2], le champ $\mathbf{E}_{\mathrm{p}}^{h}$ polarisant une molécule $h$, dépend des voisines $l$ :

$$
\mathbf{E}_{\mathbf{p}}^{h}=\left[(U)+\sum_{l \in D_{\mathrm{f}}^{h}}\left(S^{l h}\right)(\alpha)^{l}\right] \mathbf{G}^{h}
$$

$\left(S^{l h}\right)$ est la matrice qui relie le champ en $h$, créé par un dipôle en $l$, à ce dipôle :

$$
\begin{aligned}
& \underset{\substack{(i, j=x, y, z) \\
S_{i j}^{l h}}}{l}=\frac{1}{4 \pi \varepsilon_{0}} \cdot \frac{3 R_{i}^{l h} \cdot R_{j}^{l h}-\left(R^{l h}\right)^{2} \Delta_{i j}}{\left(R^{l h}\right)^{5}} \\
& \left(S^{l h}\right)=\left(S^{h l}\right)
\end{aligned}
$$

$\Delta$ est l'indice de Kronecker, $R^{\text {lh }}$ la distance mutuelle. $D_{\mathrm{f}}^{h}$ désigne l'ensemble des proches voisins de $h$, $\mathbf{G}^{h}$ le champ polarisant moyen. La molécule $h$ présente un moment dipolaire $\boldsymbol{\mu}^{h}$ induit :

$$
\begin{aligned}
\boldsymbol{\mu}^{h} & =\left[(\alpha)^{h}+(Z)^{h}\right] \mathbf{G}^{h} \\
(Z)^{h} & =(\alpha)^{h} \sum_{l \in D_{\mathrm{f}}^{h}}\left(S^{l h}\right)(\alpha)^{l}
\end{aligned}
$$

$(Z)^{h}$ peut être compris comme représentant une hyperpolarisabilité collisionnelle. Les intensités $I_{i j}(i, j=x, y, z)$ sont :

$$
\begin{aligned}
& I_{i j} \sim|\mathbf{G}|^{2}\left\langle\sum _ { h , m } \left(\alpha_{i j}^{h} \alpha_{i j}^{m}+\alpha_{i j}^{h} Z_{i j}^{m}+\right.\right. \\
& \left.\left.\quad+\alpha_{i j}^{m} Z_{i j}^{h}+Z_{i j}^{h} Z_{i j}^{m}\right) \cos \varphi^{h m}\right\rangle
\end{aligned}
$$

$\varphi^{h m}$ est le déphasage dû aux propagations, $\langle>$ indique une moyenne sur toutes les configurations. Dès que $\varphi^{h m}$ est sensiblement non nul, $(Z)^{m}$ est indépendant de $(Z)^{h}$ et de $(\alpha)^{h}$, de plus $\left\langle(Z)^{m}\right\rangle$ est nulle, on peut alors remplacer $\cos \varphi^{h m}$ par un quand il est en facteur de $\alpha_{i j}^{h} Z_{i j}^{m}$ ou de $Z_{i j}^{h} Z_{i j}^{m}$. La relation (6) devient alors :

$$
\begin{aligned}
I_{i j} \sim|\mathbf{G}|^{2}\left\langle\sum _ { h , m } \left(\alpha_{i j}^{h} \alpha_{i j}^{m}\right.\right. & \cos \varphi^{h m}+ \\
& \left.\left.+Z_{i j}^{h} Z_{i j}^{m}+2 \alpha_{i j}^{h} Z_{i j}^{m}\right)\right\rangle .
\end{aligned}
$$


Nous supposerons selon l'hypothèse (notée $\mathrm{H} 1$ et discutée en annexe) qu'il n'y a pas de corrélations notables entre d'une part l'orientation d'une molécule, et d'autre part la position relative ou l'orientation de ses voisines.

Cependant et contrairement à [1], nous tiendrons compte des corrélations entre les positions des centres des molécules voisines d'une molécule donnée, ce qui conduit à des interférences parfois considérables sur le rayonnement dû aux hyperpolarisabilités collisionnelles.

Selon $\mathrm{H} 1$, la direction $l m$ qui fixe la valeur de $\left(S^{l m}\right)$ n'est pas corrélée à l'orientation d'une molécule quelle qu'elle soit. Il s'ensuit que la nullité de $\left\langle S^{l m}\right\rangle$ entraîne celle de termes tels que $\left\langle\alpha_{i j}^{h} . Z_{i j}^{m}\right\rangle ;\left(S^{l m}\right)$ entre en effet dans le calcul de $Z$.

Les $N$ molécules $h$ sont équivalentes à une molécule particulière $a$. On en déduit :

$$
I_{i j}=N .|\mathbf{G}|^{2}\left\langle\sum_{m} \alpha_{i j}^{a} \alpha_{i j}^{m} \cos \varphi^{a m}+Z_{i j}^{a} Z_{i j}^{m}\right\rangle .
$$

Les moyennes du type

$$
\left\langle\sum_{m} \alpha_{i j}^{a} \alpha_{i j}^{m} \cos \varphi^{a m}\right\rangle
$$

pour des molécules aléatoirement orientées sont bien connues en théorie classique de la diffusion, le taux de dépolarisation est alors :

$\eta=\frac{I_{x z}}{I_{z z}}=\frac{\frac{\delta^{2}}{15}+\left\langle\sum_{m} Z_{x z}^{a} Z_{x z}^{m}\right\rangle}{\bar{\alpha}^{2} \cdot \frac{R T \chi_{\mathrm{T}}}{V}+\frac{4}{45} \cdot \delta^{2}+\left\langle\sum_{m} Z_{z z}^{a} Z_{z z}^{m}\right\rangle}$

$R T \chi_{\mathrm{T}} / V$ est le facteur intervenant dans les fluctuations de densité. Si on néglige $Z, \eta$ se réduit à la valeur qu'il aurait en l'absence de collision $\left(\eta_{\mathrm{nc}}\right)$. Pour les molécules expérimentalement étudiées $\left(\mathrm{N}_{2}, \mathrm{C}_{2} \mathrm{H}_{4}\right)$ à moyenne densité, les ordres de grandeur des différents termes ( prenant comme unité $\left.\alpha^{2} \cdot \frac{R T \chi_{\mathrm{T}}}{V}+\frac{4 \delta^{2}}{45}\right)$ sont $10^{-2}$ pour $\delta^{2} / 15,10^{-3}$ pour

$$
\left\langle\sum_{m} Z_{x z}^{a} Z_{x z}^{m}\right\rangle \text { et }\left\langle\sum_{m} Z_{z z}^{a} Z_{z z}^{m}\right\rangle
$$

on peut donc négliger l'influence de $Z$ au dénominateur de (9) et scinder l'équation (9) en deux termes :

où

$$
\eta=\eta_{\mathrm{nc}}+\eta_{\mathrm{c}}
$$

$$
\begin{aligned}
\eta_{\mathrm{nc}} & =\frac{3 \delta^{2}}{45 \alpha^{2} \cdot \frac{R T \chi_{\mathrm{T}}}{V}+4 \delta^{2}} \\
\eta_{\mathrm{c}} & =\frac{\left\langle\sum_{m} Z_{x z}^{a} Z_{x z}^{m}\right\rangle}{\bar{\alpha}^{2} \cdot \frac{R T \chi_{\mathrm{T}}}{V}+\frac{4}{45} \delta^{2}} .
\end{aligned}
$$

Nous appellerons $F$ la quantité

$$
\left\langle\sum_{m} Z_{x z}^{a} Z_{x z}^{m}\right\rangle
$$

Calcul de $\eta_{\mathbf{c}}$ à basse densité. - A basse densité, la molécule $a$ n'est perturbée que par une seule autre (notée $p$ ); $Z_{x z}^{a}, Z_{x z}^{m}$ sont indépendants sauf si $m$ désigne $p$ ou $a$. La nullité de $\left\langle Z_{x z}^{a}\right\rangle$ et de $\left\langle Z_{x z}^{m}\right\rangle$ montre que, dans $F$, seules comptent les valeurs de $m$ égales à $a$ et $p$ :

$$
F=\left\langle\left(Z_{x z}^{a}\right)^{2}\right\rangle+\left\langle Z_{x z}^{a} Z_{x z}^{p}\right\rangle .
$$

Si on développe $Z_{x z}^{a}, Z_{x z}^{p}$ et si on regroupe les termes de façon à pouvoir faire des moyennes indépendantes, on obtient :

$$
\begin{array}{r}
F=\sum_{\eta, \theta, \mu, v=x, y, z}\left[\left\langle\alpha_{x \eta}^{a} \alpha_{x \mu}^{a}\right\rangle\left\langle S_{\eta \theta}^{p a} \cdot S_{\mu \nu}^{p a}\right\rangle\left\langle\alpha_{\theta z}^{p} \alpha_{v z}^{p}\right\rangle+\right. \\
\left.+\left\langle\alpha_{x \eta}^{a} \alpha_{v z}^{a}\right\rangle\left\langle S_{\eta \theta}^{p a} S_{\mu \nu}^{a p}\right\rangle\left\langle\alpha_{\theta z}^{p} \alpha_{x \mu}^{p}\right\rangle\right]
\end{array}
$$

tenant compte des moyennes angulaires suivantes :

$$
\begin{aligned}
\left\langle\alpha_{i j} \cdot \alpha_{k l}\right\rangle & =\bar{\alpha}^{2} \Delta_{i j} \Delta_{k l}+\left(\frac{\delta}{3}\right)^{2} D_{i j k l} \\
\left\langle S_{i j} \cdot S_{k l}\right\rangle & =\frac{1}{4 \pi \varepsilon_{0}}\left\langle\frac{1}{r^{6}}\right\rangle D_{i j k l} \\
D_{i j k l} & =\frac{1}{5}\left[3 \Delta_{i k} \Delta_{j l}+3 \Delta_{i l} \Delta_{j k}-2 \Delta_{i j} \Delta_{k l}\right]
\end{aligned}
$$

où $r$ représente la distance entre $a$ et $p$. On en déduit la valeur suivante de $F$ :

$F=\left(\frac{1}{4 \pi \varepsilon_{0}}\right)^{2}\left\langle\frac{1}{r^{6}}\right\rangle\left[\frac{6}{5} \bar{\alpha}^{4}+\frac{14}{75} \bar{\alpha}^{2} \delta^{2}+\frac{44}{1125} \delta^{4}\right]$.

On retrouve bien pour $\eta_{\mathrm{c}}$, la valeur connue pour les molécules isotropes [2] en faisant $\delta=0$.

Dans le cas des molécules isotropes, les deux termes de (13) sont égaux. Cette égalité a été supposée par Trappeniers et coll., même dans le cas des molécules anisotropes, ce qui est inexact et les conduit à une autre expression, pour le terme en $\alpha^{2} \delta^{2}$ et en $\delta^{4}$ ([1], éq. (21)).

Calcul de $\eta_{c}$ à densité modérée pour des molécules peu anisotropes. - On suppose que dans (16) le terme dominant est le terme en $\bar{\alpha}^{4}$. L'erreur ainsi commise $\left(\sim \frac{7}{45} \cdot \frac{\delta^{2}}{\bar{\alpha}^{2}}\right.$ soit $\sim 2,3 \eta_{0}$, où $\eta_{0}$ est la valeur de $\eta$ à densité nulle quand $R T \chi_{\mathrm{T}} / V=1$ ) est faible dans le cas de $\mathrm{N}_{2}$ et $\mathrm{C}_{2} \mathrm{H}_{4}\left(\eta_{0}=0,99 \times 10^{-2}\right.$ et $\left.1,20 \times 10^{-2}\right)$. L'expression de $\eta_{\mathrm{c}}$ est alors valable pour une molécule de forme quelconque, pour le calcul de $\eta_{\mathrm{nc}}$, il suffit de généraliser la définition de $\delta$. Cette approximation revient à confondre $\bar{\alpha}(U)$ et $(\alpha)$ pour calculer $F$. Celui-ci devient alors :

$$
F=\bar{\alpha}^{4}\left\langle\sum_{l, m, t}^{\prime} S_{x z}^{a l} S_{x z}^{m t}\right\rangle
$$


soit

où

$$
\left.\begin{array}{c}
F=2 \bar{\alpha}^{4}\left\langle\sum^{\prime}\left(S_{x z}^{a l}\right)^{2}\right\rangle I \\
I=\frac{\left\langle\sum_{l, m, t}^{\prime} S_{x z}^{a l} S_{x z}^{m t}\right\rangle}{2\left\langle\sum^{\prime}\left(S_{x z}^{a l}\right)^{2}\right\rangle}
\end{array}\right\}
$$

l'indice (') affecté à $\sum$ indique qu'on restreint les sommes à $l \in D_{\mathrm{f}}^{a}, t \in D_{\mathrm{f}}^{m}$. Le facteur $I$ a été calculé de plusieurs manières $[2,5]$. Pour les gaz à densité modérée le calcul de Alder et coll. [5] semble le meilleur [4].

Comparaison avec l'expérience.

Une analyse détaillée est présentée en [4], que nous résumons ici. La mesure de $\eta_{0}$ permet de déterminer $\delta^{2} / \alpha^{2}$, donc, connaissant l'équation d'état, de calculer $\eta_{\mathrm{nc}}$ (11) et le dénominateur de (12). $\eta_{\mathrm{c}}$ (12) peut s'écrire :

$$
\begin{array}{rl}
\eta_{\mathrm{c}}=\frac{6}{5}\left(\frac{\bar{\alpha}}{4 \pi \varepsilon_{0}}\right)^{2} & n \int\left(\frac{1}{r^{6}} \mathrm{e}^{-W / k T} \mathrm{~d} \tau\right) \times \\
& \times L \times I \times \frac{\bar{\alpha}^{2}}{\bar{\alpha}^{2} \frac{R T \chi_{\mathrm{T}}}{V}+\frac{4}{45} \delta^{2}}
\end{array}
$$

$n$ est la densité numérique de molécules, $W$ le potentiel intermoléculaire, $L$ un facteur tenant compte d'une distribution exacte des couples représentée par la fonction de distribution radiale $g(r)$ (à haute densité $\left.g(r) \neq \mathrm{e}^{-W / k T}\right)$ :

$$
L=\left[\int \frac{g(r)}{r^{6}} \mathrm{~d} \tau / \int \frac{\mathrm{e}^{-W / k T}}{r^{6}} \mathrm{~d} \tau\right] .
$$

En introduisant la densité $\rho$ en Amagat $\left(\rho=n V_{0} / N_{\mathrm{a}}\right.$, $V_{0}$ volume molaire S.T.P., $N_{\mathrm{a}}$ nombre d'Avogadro); $\eta_{\mathrm{c}}$ ne peut se mettre sous la forme :

$$
\eta_{\mathrm{c}}=A . \xi
$$

où

$$
\begin{aligned}
A & =\frac{6}{5}\left(\frac{\bar{\alpha}}{4 \pi \varepsilon_{0}}\right)^{2} \frac{N}{V_{0}} \int \frac{\mathrm{e}^{-W / k T}}{r^{6}} \mathrm{~d} \tau \\
\xi & =\rho \times \frac{\bar{\alpha}^{2}}{\bar{\alpha}^{2} \frac{R T \chi_{\mathrm{T}}}{V}+\frac{4}{45} \delta^{2}} \times L \times I
\end{aligned}
$$

$A$ est une constante, $\xi$ dépend de la densité $\rho$. Pour calculer $\xi$ nous avons évalué $L$ et $I$ (qui restent voisins de 1) en utilisant [5] et en assimilant les molécules à des sphères rigides. Une mesure précise de $\eta$, et le calcul de $\eta_{\mathrm{nc}}$ à cette densité (11), permet de déterminer $\eta_{\mathrm{c}} \quad\left(\eta_{\mathrm{c}}=\eta-\eta_{\mathrm{nc}}\right)$. L'équation (21) permet alors de déduire une valeur expérimentale de $A$, utilisant une méthode de moindre carré. Cette valeur semble bien indépendante du domaine de densité étudié [4]. Les valeurs expérimentales ainsi obtenues pour $\mathrm{N}_{2}$ et $\mathrm{C}_{2} \mathrm{H}_{4}\left(0,9 \times 10^{-5}\right.$ et $\left.4,5 \times 10^{-5}\right)$ sont en bon accord avec les valeurs théoriques (22), calculées en prenant pour $W$ un potentiel L.J., soit $1,1 \times 10^{-5}$ et $5,2 \times 10^{-5}$. Il est vrai que la précision expérimentale est médiocre $(\sim 30 \%)$, car une faible erreur sur $\eta_{0}$ se répercute sur $\eta_{\mathbf{n c}}$ et conduit à une erreur relative importante sur $\eta_{\mathrm{c}}$.

Conclusions. - La théorie présentée ici semble assez bien justifiée pour des molécules faiblement anisotropes $\left(\eta_{\mathrm{nc}} \leqslant 1,3 \%\right)$ à densités modérées. On a tenu compte des phénomènes d'interférence dans la diffusion liée aux hyperpolarisabilités collisionnelles. Cependant, on a ignoré les modifications de polarisabilité en cours de collision, et les corrélations d'orientations moléculaires à hautes densités. Ces corrélations sont discutées par Trappeniers et coll. en [1].

Annexe. - Nous avons négligé les interférences portant sur l'intensité diffusée par les anisotropies propres des molécules, tout en mettant en évidence (18) les interférences portant sur l'intensité diffusée par l'anisotropie collisionnelle $[8,3]$. Nous allons estimer l'importance des interférences entre deux molécules d'azote distantes de $6,68 \AA$, ce qui est l'ordre de grandeur de la distance entre molécules voisines à 150 Amagats et à température ambiante. Selon un résultat connu le facteur d'interférence $I$ est alors :

$$
I_{\text {propre }}=\frac{1+3\left\langle\operatorname{Cos}^{2} \psi\right\rangle}{2}
$$

où $\psi$ est l'angle de la deuxième molécule avec la première.

Reprenant un potentiel du type Lennard-Jones à deux centres, employé par Quentrec et Brot [6], nous avons estimé l'énergie de deux molécules pour des orientations définies. La pondération par le facteur de Boltzmann de ces différentes orientations nous permet d'évaluer $I_{\text {propre }}$. Dans le cas envisagé, nous obtenons : $I_{\text {propre }}=1+5 \times 10^{-3}$ très proche de 1 .

Ceci fournit un ordre de grandeur des facteurs d'interférences dues aux corrélations d'orientation dans l'azote à 150 Amagats. Ainsi, l'effet de ces corrélations est très faible à cette densité. Ceci est confirmé par les travaux de Keijser R. A. J. [7] : il a étudié l'élargissement de la raie Rayleigh dépolarisée $(\Delta J=0)$ et des raies Raman de rotation dans différents gaz, à des pressions inférieures à $100 \mathrm{~atm}$; les élargissements, mesurables au Fabry-Perot, restent faibles, traduisant le peu d'efficacité des choses au point de vue réorientation. Pour l'azote (150 Amagats, $20^{\circ} \mathrm{C}$ ), le taux de dépolarisation propre des molécules est de l'ordre de $1,8 \times 10^{-2}$, affecté par un facteur d'interférence 1,0055 , tandis que la contribution collisionnelle, calculée en négligeant les interférences, est de l'ordre de $1,4 \times 10^{-3}$, affectée d'un facteur d'interférence 0,5 . On note que l'effet des interférences est environ 10 fois plus important sur la deuxième contribution que sur la première. 


\section{Bibliographie}

[1] Trappeniers, N. J., Kuz, V. A. et Ten Seldam, C. A., Physica 57 (1972) 294.

[2] Thibeau, M., Oksengorn, B. et Vodar, B., J. Physique 29 (1968) 287.

[3] Sung Chung, An. Thèse, Catholic University of Washington (1975).
[4] Berrue, J., Chave, A., Dumon, B. et Thibeau, M., J. Physique (à paraître).

[5] Alder, B. J., Weis, J. J. et Strauss, H. L., Phys. Rev. A 7 (1973) 281.

[6] Quentrec, B. et Brot, C., Phys. Rev. A 12 (1975) 272.

[7] KeIJSER, R. A: J., Thèse Amsterdam (1973).

[8] Thibeau, M., Thèse Paris No A04302 (1970). 